\title{
Electronic properties of magnetically doped nanotubes
}

\author{
KEIVAN ESFARJANI*, Z CHEN ${ }^{\dagger}$ and Y KAWAZOE ${ }^{\dagger}$ \\ Sharif Institute of Technology, and Institute for Physics and Mathematics, Tehran, Iran \\ 'Institute for Materials Research, Tohoku University, Sendai, Japan
}

\begin{abstract}
Effect of doping of carbon nanotubes by magnetic transition metal atoms has been considered in this paper. In the case of semiconducting tubes, it was found that the system has zero magnetization, whereas in metallic tubes the valence electrons of the tube screen the magnetization of the dopants: the coupling to the tube is usually antiferromagnetic (except for $\mathrm{Cr}$ ).
\end{abstract}

Keywords. Carbon nanotubes; magnetic doping; electronic properties; ab initio.

\section{Introduction}

Due to their interesting electrical, mechanical and thermal properties, carbon nanotubes have attracted the attention of many research groups and created a large body of publications since their discovery in 1991 (Iijima 1991). Recent experimental (Lee et al 1997; Rao et al 1997; Grigorian et al 1998a, b) and theoretical (Miyamoto et al 1995; Esfarjani et al 1999) studies on doping nanotubes focused on doping by alkali metal or halogene elements as electron donors or acceptors, respectively. The role of magnetic dopants has not been considered however.

\section{Method}

In this work, we have computed by first-principle methods the electronic properties of both metallic and semiconducting nanotubes doped with transition metal atoms: $\mathrm{Cr}, \mathrm{Mn}, \mathrm{Fe}, \mathrm{Co}$ and $\mathrm{Ni}$. The program used is VASP (Kresse et al 1996), which uses plane-wave and Furthmüller bases with ultra-soft pseudopotentials within the spin-polarized generalized gradients approximation (GGA). The unit cell dimensions were $12.5 \times 12.5 \times$ $4.92 \AA$ for the $(4,4)$ armchair $(4,4)$, and $12.5 \times 12.5$ $\times 4.26 \AA$ for the $(7,0)$ zigzag tube, respectively. A cutoff energy of $358.4 \mathrm{eV}$ and $21 \mathrm{k}$ points along the tube axis were used to assure convergence of the total energy results to within $10 \mathrm{meV}$.

\section{Results and discussion}

As shown in table 1, the formation energy of doped tubes is positive meaning that magnetic transition metal elements do not like to enter the nanotubes. This forma-

\footnotetext{
*Author for correspondence
}

tion energy is computed from the difference between the total energy of a relaxed tube with one atom per two unit cells, and the sum of the total energies of a relaxed empty tube and a relaxed atomic chain of transition metal element. Since the width of the unit cell is too small for a single transition metal element, the single atom was put in two unit cells. One could also fit two atoms into two unit cells for tubes of larger radius, but calculations became prohibitive at this level. Note that a positive formation energy only implies that a doped tube is in a metastable state.

From the comparison of the formation energies, we find that among the $(4,4)$ tubes, the Co-doped tube is thermodynamically unstable, and among the $(7,0)$ tubes, Mn doping makes it unstable. The effect of the dopant is usually to add $d$-levels to the band structure of the undoped tube. These levels are shifted with respect to each other, and that is reflected in the density of states (DOS) of up-spin and down-spin states. The Fermi energy is usually located between the two split levels and is slightly larger than that of the undoped tube, meaning that there is charge transfer from dopant atoms to the tube. This charge is found to be about 1 electron per dopant atom. By calculating the area under the DOS of the undoped tube between the Fermi energies of the undoped tube and that of the doped tube, one can estimate the number of extra electrons on the tube. If the Fermi level lies in the middle of a up-spin $d$ band, then

Table 1. Formation energy and magnetization of doped $(4,4)$ nanotubes.

\begin{tabular}{|c|c|c|c|c|}
\hline & $E_{\mathrm{F}}(\mathrm{eV})$ & Doped tube $\left(\grave{\imath}_{\mathrm{B}}\right)$ & Chain $\left(\grave{1}_{\mathrm{B}}\right)$ & ÄMag $\left(\grave{i}_{\mathrm{B}}\right)$ \\
\hline $\mathrm{Cr}^{-}$ & 0.7916 & 4.9367516 & 3.9047658 & 1.0319858 \\
\hline $\mathrm{Mn}^{-}$ & $0 \cdot 3651$ & 2.5339235 & $4 \cdot 1394122$ & -1.6054887 \\
\hline $\mathrm{Fe}^{-}$ & $0 \cdot 3707$ & $-2 \cdot 0973932$ & 3.9987493 & -6.0961425 \\
\hline $\mathrm{Co}^{-}$ & $0 \cdot 6702$ & 1.0833886 & 1.7334098 & -0.6500212 \\
\hline $\mathrm{Ni}^{-}$ & $0 \cdot 3136$ & $0 \cdot 0000653$ & $2 \cdot 0256558$ & $-2 \cdot 0256558$ \\
\hline
\end{tabular}



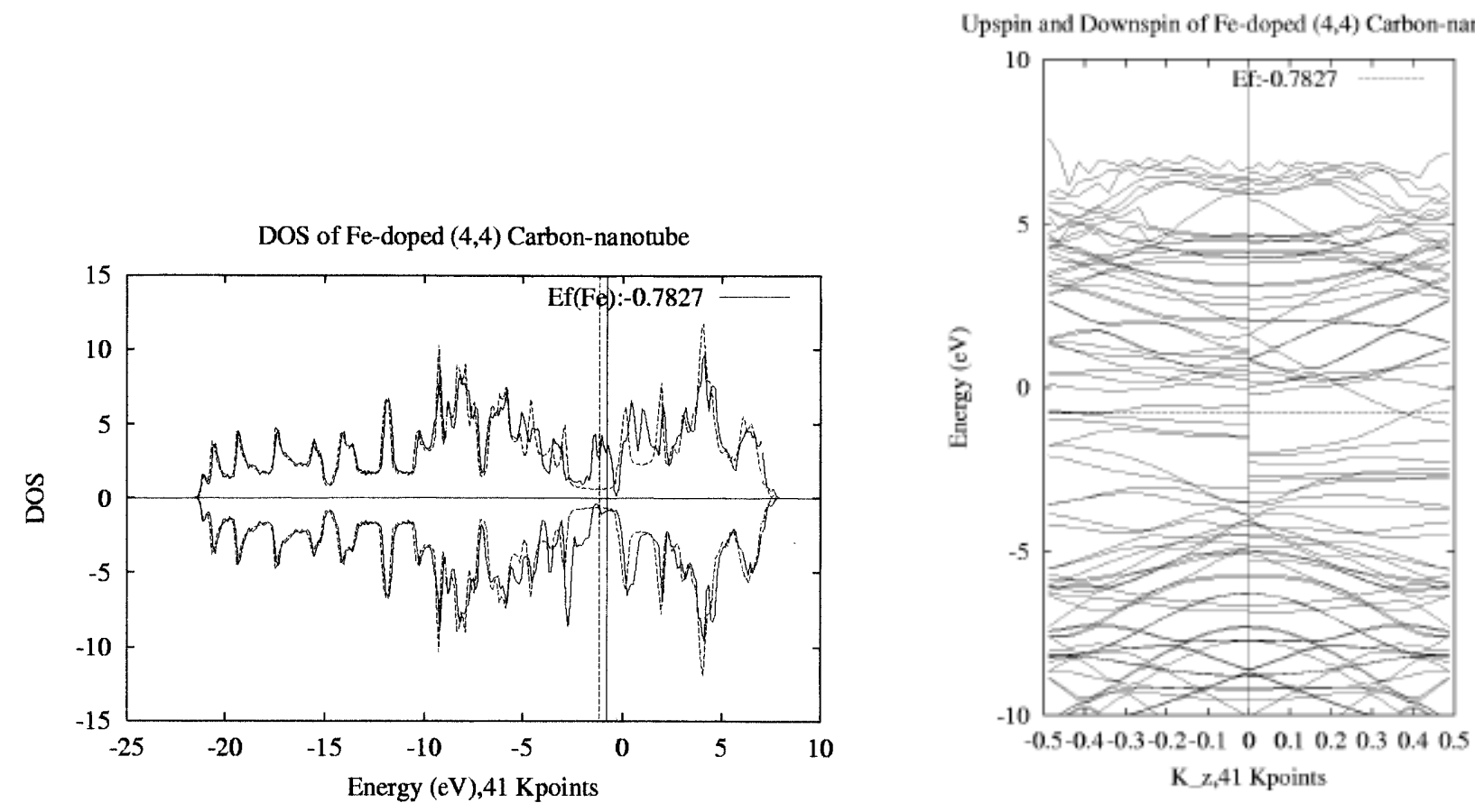

Figure 1. DOS (left) and band structure (right) of Fe-doped $(4,4)$ nanotube.
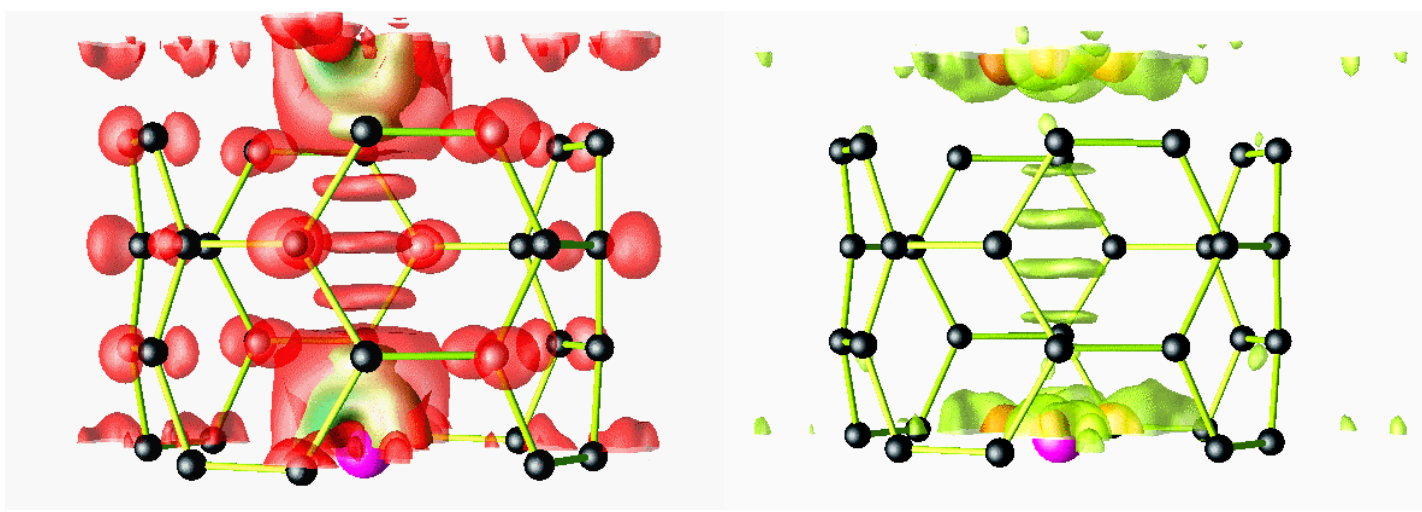

Figure 2. Up-spin and down-spin electron density of the Co doped $(4,4)$ nanotube.

the DOS of up spin states at the Fermi level would be larger than that of the down-spin states. This can be seen in figure 1 for the Fe-doped $(4,4)$ tube. This has important consequences regarding the functionality of this system as a spin valve device.

There is also hybridization between the $d$ levels and the nanotube conduction bands. The $\mathrm{Fe}$ atom for instance, when doped inside the tube, moves away from the centre and attaches itself to the wall. Due to this strong overlap of $d$ orbitals of $\mathrm{Fe}$ and $\pi$ orbitals of carbon, mixing and hybridization occurs. The relaxation of the atom away from the axis of the tube occurred for $\mathrm{Mn}$ and $\mathrm{Fe}$ atoms in the $(4,4)$ armchair tube. The $\mathrm{Ni}$ and $\mathrm{Co}$ atoms stayed on the tube axis after relaxation.

Next, we focus on the magnetization distribution in the system. In the case of the zigzag tube, it was found that there is no magnetization present. For metallic tubes, however, there is a net magnetization weaker than its atomic value for the dopant, except for Cr-doped tubes where the magnetization is enhanced compared to the atom. This was the only instance of ferromagnetic coupling between the tube and transition metal element. For other doping elements, conduction states of the metallic tube screen out the magnetization of the dopant by hav- 
ing their electrons' spins line up antiparallel to that of the $d$ electrons. The induced magnetization on the tube is, however, an order weaker than that of the dopant atom. The other interesting finding is that $\mathrm{Ni}$ becomes nonmagnetic although the Fermi level lies in the middle of the $d$ band. No exchange splitting is seen for the $d$ levels of $\mathrm{Ni}$ as its $d$ levels are almost filled. As an illustration of the magnetization distribution, figure 2 shows the density of up-spin electrons on the left and down-spin electrons on the right for the Co-doped $(4,4)$ tube.

\section{Acknowledgement}

The authors would like to thank the staff of the Computational Materials Science Centre, Institute for Materials
Research, Japan, for the maintenance of the HITAC supercomputing system.

\section{References}

Esfarjani K, Farajian A A and Kawazoe Y 1999 Appl. Phys. Lett. 7479

Grigorian L et al 1998a Phys. Rev. B58 R4195

Grigorian Let al 1998b Phys. Rev. Lett. 805560

Iijima S 1991 Nature 35456

Kresse G and Furthmüller J 1996 Phys. Rev. B54 11169 (VASP was developed at the technical University of Vienna; see also the following link: http://cms.mpi.univie.ac.at/vasp)

Lee R S et al 1997 Nature $\mathbf{3 8 8} 255$

Miyamoto Y et al 1995 Phys. Rev. Lett. 742993

Rao A M et al 1997 Nature $\mathbf{3 8 8} 257$ 